\title{
Over-tube Apparatus for Increasing the Capabilities of an Articulated Robotic Probe
}

\author{
Amir Degani, Stephen Tully, Brett Zubiate and Howie Choset
}

\begin{abstract}
This video elaborates on a new active and controllable over-tube addition to the highly articulated robotic probe; the HARP. This over-tube allows the current HARP mechanism to double its overall length and allows it to perform more complex tasks. We explain the design concept of the current HARP and the novel over-tube mechanism and show two proof-of-concept experiments demonstrating the use of the active over-tube.
\end{abstract}

\section{INTRODUCTION}

At Carnegie Mellon University and the University of Pittsburgh, we have been developing a small surgical snake robot which we term the HARP; highly articulated robotic probe [1], [5], [4]. The HARP can use its many degrees of articulation to reach anatomical targets deep in the body without disturbing surrounding tissues, which of course is key for minimally invasive surgery.

We are now interested in natural orifice surgery, which undoubtedly requires a longer articulated probe. We therefore began development of an over-tube for natural orifice translumenal endoscopic surgery (NOTES). The current video illustrates the use of a novel over-tube to increase the HARP's capabilities and to enable it to reach places it could not before.

\section{RELATED WORK}

Minimally invasive surgery (MIS) is now known to have many benefits over traditional surgery. These benefits include reduced pain, faster healing and recovery, and fewer complications due to less tissue disruption [2], reduced blood loss and decreased post-operative pain [3]. The most known and widely accepted device is the da Vinci system (Intuitive Surgical Inc., Sunnyvale, CA) which uses conventional industrial robot arms that grab custom designed laparoscopic tools with which MIS can be performed. The main disadvantage of these current robotic mechanisms is the use of rigid endoscopic tools. Classic flexible endoscopic tools exist, however, the lack of control reduces their ability to perform complicated manipulation tasks.

A. Degani is with the Faculty of Civil and Environmental Engineering, Technion - Israel Institute of Technology, Haifa 32000, Israel adegani@technion.ac.il

S. Tully is with the Electrical Engineering Department, Carnegie Mellon University, 5000 Forbes Avenue, Pittsburgh, PA, USA stullydece.cmu.edu

B. Zubiate is with Medrobotics bzubiatte@medrobotics.com

$\mathrm{H}$. Choset is with the Robotics Institute, Carnegie Mellon University, 5000 Forbes Avenue, Pittsburgh, PA, USA chosetecs. cmu.edu

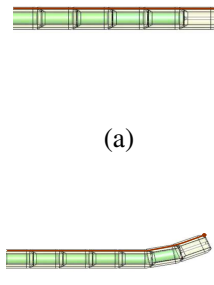

(d)

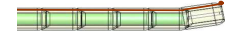

(b)

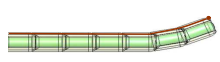

(e) (c)

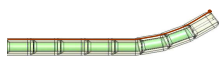

(f)

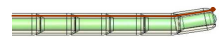

Fig. 1. Typical gait sequence of the HARP mechanism.

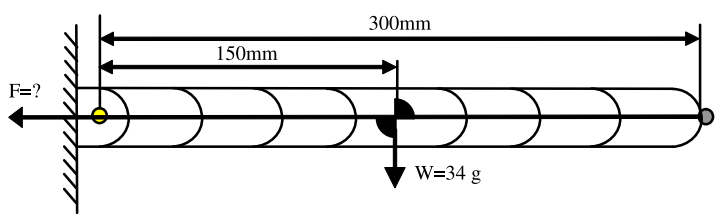

(a)

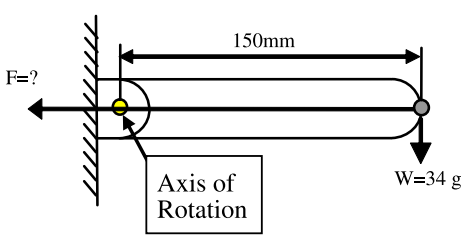

(b)

Fig. 2. Schematic of extreme cantilever configuration. 2(a) - extreme configuration. 2(b) - Simplified model.

\section{CURRENT HARP DESIGN AND OPERATION}

A novel feature of the HARP is that it uses conventional motors. The HARP's design consists of two concentric tubes. Each tube comprises many plastic links strung together on strings. The two tubes can alternate between being rigid or limp, while the outer one can also direction the distal link. The HARP follows an arbitrary curve in three dimensional space by alternating the rigidity/limpness of the outer and inner mechanisms. See Fig. 1 for the gait sequence of the HARP.

An extreme or worst-case configuration of the current HARP (without the proposed over-tube) is shown in Fig. 2(a). This worst-case configuration occurs when the device is stretched out in a cantilevered position, the outer mechanism is limp, and the inner mechanism supports its own weight as well as the weight of the outer mechanism. It is easy to see that because the force required by the strings to be pulled is proportional to the weight of the HARP, its length is limited. 


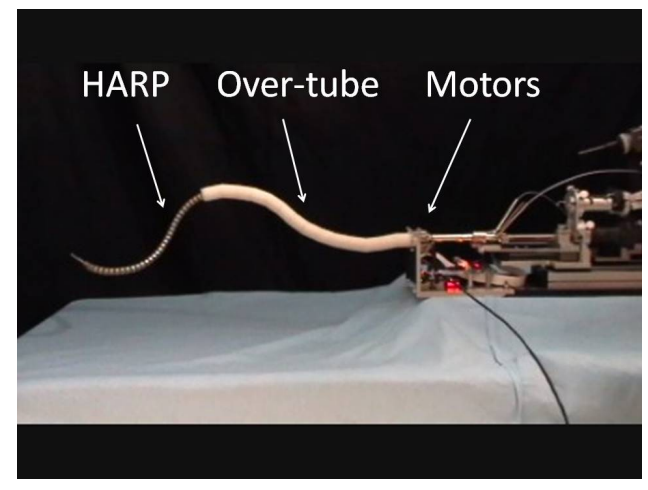

Fig. 3. The HARP mechanism with the over-tube.

\section{OVER-TUBE DESIGN AND OPERATION}

The over-tube that we are developing allows a dramatic increase of the mechanism's length. This over-tube is a third concentric tube controlled by three motors (Fig. 3). Once the over-tube is driven passively into its configuration, the strings are tightened and the over-tube becomes a rigid support cannula for the HARP. By including this third tube we can double the overall length of our probe.

We begin the use of the HARP with the over-tube by first making the three concentric tubes limp. By being limp, the entire mechanism can be inserted into a natural orifice. We can also slightly articulate the over-tube by using its three motors. The visualization instrument is onboard the middle tube which is distally flush to the over-tube in this part of the procedure. By using the visualization instrument, the operator decides when to stop the insertion of the mechanism and tightens and locks the over-tube in place. From now on the distal part of the over-tube becomes the proximal port for the HARP to begin the articulation.

\section{PROOF-OF-CONCEPT DEMONSTRATIONS}

To motivate the use of the over-tube with the HARP, we have conducted two proof-of-concept demonstrations. The first is a bench-top demonstration of a $600 \mathrm{~mm}$ HARP mechanism with and without the help of the over-tube. Fig. 4(a) shows the long, $600 \mathrm{~mm}$ HARP failing under its own weight while Fig. 4(b) shows the HARP successfully maneuvering using the over-tube mechanism. The mechanism can first be passively shaped into position imitating the insertion of the over-tube into a natural orifice. Then the probe exits the overtube and the articulation begins.

The second demonstration shows the possibility to use this mechanism in domains other than the medical field. In Fig. 4(c) we insert the HARP with the over-tube into a mobile robot simulating a suspicious vehicle. We first drive the probe into a hole on the vehicle. We reduce the rigidity of the overtube to allow the mechanism to passively enter the vehicle. After the over-tube is sufficiently inserted into the vehicle, we rigidify it and start the articulation of the HARP. Fig. 4(d)

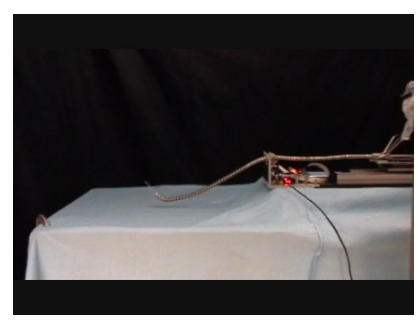

(a)

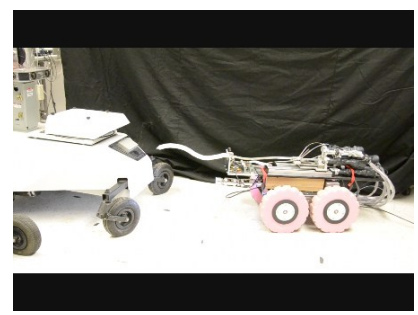

(c)

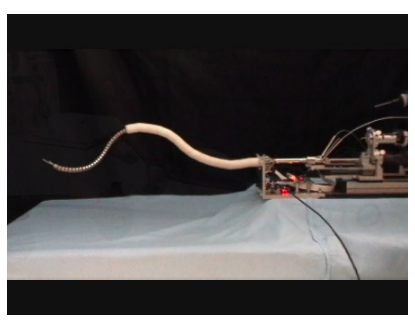

(b)

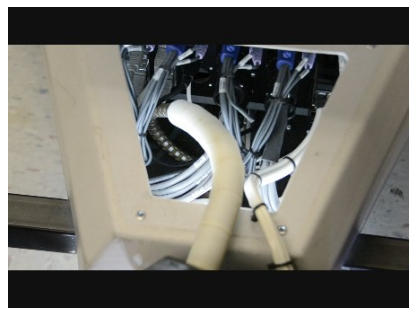

(d)
Fig. 4. Proof-of-concept demonstration of the HARP mechanism with the over-tube. 4(a) - bench-top demonstration of a $600 \mathrm{~mm}$ HARP mechanism failing without the use of the over-tube. 4(b) - bench-top demonstration of a $600 \mathrm{~mm}$ HARP mechanism supported by the over-tube. 4(c) - Vehicle inspection with the HARP and over-tube. 4(d) - Final configuration of the probe inside the vehicle being inspected.

shows the final configuration of the HARP inside the cavity of the mobile robot.

\section{CONCLUSIONS AND FUTURE WORKS}

This video shows the addition of an active and controllable over-tube to the HARP mechanism which allows the mechanism to double its overall length while keeping its simplicity. Our current goal is to bring our mechanism to the operating room to use in NOTES procedures. The use of our over-tube is adequate to NOTES procedure since the HARP can be passively guided into a natural orifice and only then articulated to perform the specific task.

\section{ACKNOWLEDGMENTS}

The authors would like to thank Michael Schwerin, Cornell Wright, Shyam Thakkar, and Michael Awad.

\section{REFERENCES}

[1] A. Degani, H. Choset, A. Wolf, T. Ota, and M. A. Zenati. Percutaneous intrapericardial interventions using a highly articulated robotic probe. In The First IEEE/RAS-EMBS International Conference on Biomedical Robotics and Biomechatronics, (BioRob'06), 2006.

[2] T. J. Huang, R. W. Hsu, S. H. Chen, and Y. Y. Lee. Minimal access surgery in managing anterior lumbar disorders. Clin Orthop Relat Res, (387):140-7, 2001.

[3] S. M. Nymberg and A. H. Crawford. Video-assisted thoracoscopic releases of scoliotic anterior spines. AORN journal, 63(3):561-2, 5659; 571-5; quiz 576, pass, 1996.

[4] T. Ota, D. A. Degani, Schwartzman, B. Zubiate, J. McGarvey, H. Choset, and M. A. Zenati. A highly articulated robotic surgical system for minimally invasive surgery. The Annals of Thoracic Surgery, 87:1253-1256, 2009.

[5] T. Ota, A. Degani, B. Zubiate, A. Wolf, H. Choset, D. Schwartzman, and M. A. Zenati. Epicardial atrial ablation using a novel articulated robotic medical probe via a percutaneous subxiphoid approach. Innovations, 1(6):335, 2006. 